\title{
Diagnostic Utility Of Various Biomarkers For Prostate Cancer: A Review
}

\author{
Beenish Hussain Nomani, Mohiuddin Alamgir
}

\begin{abstract}
:
Prostate carcinoma is one of the most widespread occurring cancers in males and is the second most common cause of cancer related mortality in men around the world. The therapeutic success rate for prostate cancer can be greatly improved if the disease is detected at an early stage. Therefore, a successful therapy depends immensely on the clinical indicators (biomarkers) for early diagnosis and progression of the disease, as well as the prognosis after the clinical intervention. Despite of its limitations, prostate biopsy is the "gold standard" for diagnosis of prostatic carcinoma. Beyond the shadow of doubt, the advent of PSA level has marked a new era for the efficient screening of prostatic lesions, but PSA alone cannot be considered as an authentic tool for diagnosis. Therefore, the use of new imaging techniques and molecular markers are of great importance for an accurate diagnosis of prostate cancer. Recent advances in molecular techniques have provided new tools facilitating the discovery of new biomarkers for prostate cancer. The aim of this review is to examine the current status of prostate cancer biomarkers, with special focus the on their diagnostic utility and therefore determine a panel of two or three markers for the prompt diagnosis of prostatic carcinoma.
\end{abstract}

Keywords: biomarker, risk factor, prostate cancer, screening of prostate cancer

\section{INTRODUCTION:}

Prostate, lung and colorectal carcinoma comprise $42 \%$ of all cases reported in men, with 1 in every 5 case being that of prostate cancer. Approximately, 164,690 new cases of prostatic carcinoma were reported in the year 2018 in United States of America causing an estimated number of 29,430 deaths in men. In the last ten years, the overall incidence of cancer in men has decreased by $2 \%$ annually, with a remarkable decline in prostatic carcinoma incidence of approximately $10 \%$ every year from 2010 to 2014 . This decline in the incidence of prostatic carcinoma is accredited to effective screening measures by monitoring PSA levels in elderly men. ${ }^{1}$ According to the annual cancer registry report ,published by ShaukatKhanum Memorial Cancer Hospital and Research Centre, prostate cancer is the second most commonly occurring malignancy in Pakistani men.,

\section{Risk Factors:}

Several risk factors might be involved in the carcinogenesis; these comprise of men above the age of 50 years, androgens, genetic factors, environmental factors, family history, high fat diet, alcohol consumption, cigarette smoking and acquired somatic mutations ${ }^{4,5}$. Androgens are of paramount importance. Carcinoma of the prostate does not develop in males castrated before puberty, which strongly suggests that androgens somehow provide the "soil," the cellular context, within which prostate cancer develops. Environment moreover plays a necessary role as validated by the fact that in Japanese

M.Phil Student Beenish Hussain Nomani,
Senior Lecturer, Department of Pathology
Bahria University Medical and Dental College
Email: beenishzohaib@hotmail.com
Mohiuddin Alamgir,
Professor and HOD, Department of Pathology
Bahria University Medical and Dental College
Received: 07-02-19 emigrants travelling to United States of America, the incidence of this lesion increases. It is not prevalent within Asian population and is most frequent amid blacks and in the Scandinavian countries .Genetic factors also play a pivotal role, as there is a greater threat among first-degree family members of patients suffering from prostate tumor.

Acquired somatic mutations, comparable to other carcinomas, are the real drivers of cellular transformation.TMPRSS2ETS fusion genes are found in about $40 \%$ to $50 \%$ of prostate cancers; it is assumed that the unregulated over expression of ETS transcription factors intervenes in the differentiation of prostatic epithelial cell. ${ }^{4}$ Dietary factors include an increased intake of whole milk and dairy products is associated with aggressive prostate cancer lesions. ${ }^{6}$ Likewise; an increased consumption of a high fat diet may also lead to the development of prostatic carcinoma. ${ }^{7}$

The human prostate gland is about the size of a walnut, positioned below the urinary bladder surrounding the urethra; it is approximately 30 centimeters in length and about 20 grams in weight, composed of glands with double layer, a flat basal cell layer and an overlying columnar secretory cell layer. The prostatic stroma consists of a mixture of smooth muscles and fibrous tissue. The prostate gland can be divided into various biologically distinct regions, of which the peripheral and transition zones are of prime importance. The peripheralzone makes up about $70 \%$ of the glandular prostate. Almost $70 \%$ to $80 \%$ of the carcinomas occur in this peripheral zone, whilehyperplasticlesions are most likely to be found in the inner transition zone. ${ }^{8,9}$

Prostatic carcinomas can be classified into two major groups: (1) carcinoma of large ('primary') ducts (2) adenocarcinoma of peripheral ('secondary') ducts and acini. This morphologic distinction has conservatively been based on the belief of a different site of origin for the two tumors. However, two patterns are sometimes seen together in the same tumor, and it is believed the two types may coexist in the same prostate 
as anatomically separate lesions. Therefore, it is assumed that it is the site of the growth rather than the originthat governs the tumor architecture. ${ }^{9}$

Androgens have a vital role in the stimulation of the prostatic cancer cells, which includes testosterone and dihydrotestosterone (DHT). Majority of the androgens are secreted by the testicles, whereas the adrenal glands also produce a minute amount of male sex hormones mainly () and . Prostate is responsible for converting testosterone into dihydrotestosterone (DHT). Alterations in the androgen receptors are responsible for the development of prostate cancer. $^{10}$

Prostate cancer can be classified into various histological types; these comprise of acinaradenocarcinoma, ductal adenocarcinoma, transitional cell, squamous cell and small cell carcinoma.The Gleason Score is the grading system is utilized to determine the grade of the cancer. This system helps the clinician to select the effective treatment modality for this lesion. The most frequent sites of metastatic prostate carcinoma are lymph nodes and bone. Metastatic prostate carcinoma to the lung usually takes the form of multiple small nodules or diffuse lymphatic spread rather than large metastatic deposits. ${ }^{11,12,13}$

The clinical diagnosis of carcinoma of the prostate can be extremely difficult on prostate needle biopsy alone, and therefore the advent of immunohistochemistry has revolutionized the diagnosis of prostatic cancer. Prostatic carcinoma is developed due to various quantitative and structural genetic alterations. A number of genes have been involved in prostate cancer pathogenesis these include, ELAC2 (HPC2), MSR1, and RNASEL (HPC1) genes that have germ line mutations in prostate cancer; AR, ATBF1, mitochondria DNA, p36, PTEN, RAS BRCA1, BRCA2,, CYP17, CYP1B1,NKX3.1,CDKN1B(p27), and MYC. In this context, there is gain of function mutation as seen in AMACR and loss of function mutation as depicted by NKX3.1 AND p63. More genes relevant to prostate cancer remain to be appropriately analyzed these groups. Recognition and classification of these genes will be crucial for ameliorating the prompt diagnosis and management of this disease..$^{14,15,16}$

Certain protective dietary factors have been recognized these include consumption of the isoflavonoidgenistein found in many cruciferous vegetables, family Brassicaceae such as cauliflower, cabbage, broccoli, brussels sprouts, legumes, lycopene present in tomatoes, and inhibitor of cholesterol biosynthesis (statin drugs). ${ }^{17}$

PSA levels and digital rectal examination (DRE) have been consistently used for the screening of prostate cancer. The DRE emphasizes on volume and consistency of the prostate gland. A major number of tumors originate in the peripheral zone and are easily palpable on DRE. Carcinomas are generally hard, nodular, and irregular. ${ }^{18} \mathrm{About} 25 \%$ of males having positive findings on DRE have more chances of being diagnosed as prostatic cancer. PSA testing is a comparatively economical technique and has more individual compliance. The prime benefit of this test is its remarkable sensitivity and a major drawback is its low specificity since some pathological conditions such as benign prostatic hyperplasia, acute and chronic prostatitis can also exhibit abnormally raised PSA levels. Such false-positive results in over diagnosis leading to further evaluation for confirmation of the diagnosis, causing an increased expenditure and further utilization of invasive procedures ${ }^{19,20}$

A biomarker is described as, "a characteristic that can be measured objectively and evaluated as an indicator of normal physiological processes, pathological processes or pharmacologic responses to a therapeutic intervention" This definition explains the role of the biomarker, which includes population screening, diagnosis and prognosis. ${ }^{21}$ Disease biomarkers may vary in their types and include visual inspection (e.g, blood in urine); biochemical, enzymatic, spectrometric, or immunological measurements; and molecular changes. The hypothesis behind the use of biomarkers is that a measurement that can be used as an indicator of a physiological process and as a proxy that whether a particular pathology is existent or not. ${ }^{22}$

\section{METHODOLOGY:}

A systematic search was performed using the PUBMED NCBI database. Forty two relevant publications were selected from this literature search. Recent studies were analyzed for the use of immunohistochemistry and other techniques in the early detection and diagnosis of prostate cancer. The articles were analyzed and methodologically assessed and a review article was composed to assess the basis of diagnostic utility of various markers used for the detection of prostate cancer.

\section{LITERATURE REVIEW:}

\section{Important Biomarkers For The Diagnosis Of Prostate Cancer:}

\section{AMACR (alpha-methylacyl-CoAracemase):}

AMACR (alpha-methylacyl-CoAracemase), is an enzyme which is localized to both mitochondria and peroxisomes. It is responsible for the beta oxidation of branched fatty acids, is especially over expressed in prostatic carcinoma. Increased expression of AMACR in the premalignant lesions i.e prostatic intraepithelial neoplasia tends to potentiate the risk of adenocarcinoma of prostate. ${ }^{23}$ Alpha-methyl acylCoA racemase AMACR has been routinely over expressed in prostate cancer epithelium of premalignant and malignant lesions, hence it becomes an important definitive biomarker prostate cancer. ${ }^{24}$

\section{NKX3.1:}

NKX3.1 gene is located on chromosome 8p21, plays an important role in normal prostate development, regulating 
proliferation of glandular epithelium and in the formation of ducts in prostate. It binds to androgen receptor at the ERG gene breakpoint blocking the contact of the TMPRSS2 and ERG gene loci; preventing their recombination. ${ }^{25} \mathrm{NKX} 3.1$ promotes the recruitment of cellular proteins that initiate homology-directed DNA repair. Absence of NKX3.1 results in the accumulation of the ERG gene breakpoint which leads to error-prone non homologous end-joining. Genetic studies carried on malignant lesions of prostate gland reveal that loss of NKX3.1 is positively correlated with TMPRSS2-ERG rearrangement. ${ }^{26} \mathrm{NKX} 3.1$ is seen to be under expressed in majority of high-grade prostate carcinomas and loss of expression is found in approximately $65 \%$ to $78 \%$ of metastatic prostate carcinomas. ${ }^{25,26}$

\section{p63:}

The prostate gland is made up of two main epithelial cell types; the luminal and basal cells. The luminal cells are columnar; secrete prostate-specific antigen and specific markers, namely NKX3.1. Prostatic basal cells play an essential part in maintaining integrity of the ducts and survival of luminal cells; they express markers; p63 and cytokeratin. ${ }^{28} \mathrm{p} 63$ is an analogue of the tumor suppressor gene $\mathrm{p} 53$, which is necessary for formation of squamous epithelium. It is over expressed in normal prostate epithelial cells and is down-regulated in the presence of malignant lesion. Loss of the expression of p63 is strongly associated with the progression from benign tumors to carcinomas. ${ }^{29}$

\section{Transforming growth factor- $\beta 1$ (TGF- $\beta 1$ ):}

TGF- $\beta 1$ is a family of polypeptides which is composed of a group of cytokines that take part in the various steps of tumorogenesis and increased levels of TGF- $\beta 1$ have been seen in patients of several malignancies. ${ }^{30}$. Specific immunohistochemical studies have demonstrated overexpression of TGF- $\beta 1$ in neoplastic lesions as to the normal prostate tissue. ${ }^{31} \mathrm{H}$

igher levels TGF- $\beta 1$ have been recorded in aggressive prostate carcinoma. Various studies reported that these levels were markedly raised in lesions of prostate cancer shown to have extraprostatic expansion involving the seminal vesicle and distant metastasis to lymph nodes. ${ }^{32}$

\section{Glutathione S-transferase P1 (GSTP1):}

This class of ubiquitous multifunctional enzymes is responsible for the conjugation of reactive substances with reduced glutathione (GSH) and detoxifying them. They play an important role in protecting the cells from free radical injury, and thereby up regulated in such conditions signify them as an excellent biomarker. ${ }^{33}$ GSTP 1 methylation is frequently associated with tumor development or poor prognosis in a variety of tumors including neuroblastoma, hepatocellular carcinoma, breast, endometrial and prostate cancers. It is involved in the initial process of tumorogenesis in prostate cancer and its methylation has been inordinately researched. Different studies have revealed that about $70-80 \%$ of prostate cancer cases are methylated, whereas, cases hyperplasia of prostate are usually seen to be hypomethylated. ${ }^{34}$ PCR analysis done in some studies revealed the presence of methylated GSTP1 in the urine samples of men that underwent prostatic biopsy. ${ }^{33,34}$

\section{Chromogranin A:}

Chromogranin $\mathrm{A}$ is an acidic protein is a member of the granin family of proteins. It has been identified in all neuroendocrine cell types studied and is secreted in greater quantities than all other proteins synthesized by such cells. It is also called secretory protein I, which is encoded by the CHGA gene in human beings. The growth of prostate cells has been found to be regulated by peptides derived from chromogranin A. It has been analyzed as a marker for the diagnosis of prostate cancer. ${ }^{3}$

\section{INTERLEUKIN-6:}

Interleukin 6 is a cytokine produced by various types of cells and mediates distinct physiologic phenomena which includes; immune responses, cellular proliferation and their differentiation. Under normal circumstances, IL-6 levels in cells are nominal, although a number ofsignals mediate the marked expression of IL-6; for instance, the acute inflammatory responses to certain infections, for the activation of acute phase proteins etc. Elevated systemic levels of IL6 have been correlated with diseases such as autoimmune disorders e.g hepatitis, arthritis, Crohn's disease, ulcerative colitis, pancreatitis, and various tumors. ${ }^{36}$ Studies utilizing immunohistochemistry have elucidated that levels of IL-6 were 18 folds increased in localized prostate cancer tissue. ${ }^{37}$

\section{EPCA:}

Early prostate cancer antigen (EPCA) is a nuclear matrix protein which is associated with prostate cancer. EPCA expressed only in the patients of prostate cancer, absent in the normal prostatic tissue. Furthermore, previous studies have evaluated that EPCA could aid in the diagnosis of carcinoma of prostate even at the initial phase of carcinogenesis. ${ }^{38}$ Two EPCA subtypes have been recognized, the first one is termed as EPCA, and the second one is known as EPCA-2. Various studies have also revealed the possible benefits of EPCA-2 levels in blood samples of prostate cancer patients for clinical management of this lesion. ${ }^{39}$

\section{PTEN:}

The phosphatase and tensin homolog gene (PTEN) is situated on chromosome 10q23.3 and is localized to the mitochondria. It plays an essential role in cell cycle regulation and cell death by means of apoptosis, heavily regulated by posttranslational modifications, which includes oxidation, ubiquitination and aceylation. ${ }^{40}$ It is a negative regulator of the PIK3/AKT survival pathway and is the most commonly tumor suppressor gene in prostate cancer. PTEN expression and function, including transcriptional and post-transcriptional 
regulation, post-translational modifications, and protein-protein interactions, have been shown to be altered in human prostate cancer. ${ }^{41}$ PTEN is either lost or mutated in $50-80 \%$ of primary prostate cancer in majority of cases. ${ }^{42}$ Despite some advancement, several limitations still exist with the current technology that hinders the discovery and development of new biomarkers for all forms of cancer including prostate cancer. A critical point that has been reiterated is the fact that an ideal biomarker has to show a high level of specificity and sensitivity to prevent falsepositive screening tests, which will create anxiety in patients and lead to more expensive and invasive testing. Therefore, combining markers is thought to be the next best thing to improve the accuracy of diagnosing, treating, and surveillance of recurrence of prostate cancer.

\section{CONCLUSION:}

It was conculded that the increasing importance of biomarkers in screening and diagnosis of prostate cancer to reduce invasive follow-up procedures, help clinicians and pathologists for a prompt diagnosis and treatment. The application of biomarkers to prostate cancer is at the forefront of the research field because of the distinctive relationship between the genomic changes in the cancer cells and the disease progression.

\section{REFERENCES:}

1. Siegel RL, Miller KD, Jemal A. Cancer statistics, 2018. CA Cancer J Clin 2018;68(1):7-30. Available from: http://doi.wiley.com/10.3322/caac.21442

2. Mahmood S, Faraz R, Yousaf A, Asif H, Atif A, Waseem M, et al. Annual cancer registry report-2016, of the shaukatkhanum memorial cancer hospital \& research center, pakistan. 2016

3. Beg BM, Pasha AB, Butt NF, Qureshi SS, Randhawa FA. Prostate Cancer Awareness And Knowledge; A Study Of Adult Men In Lahore, Pakistan. Pakistan Journal of Public Health. 2018;8(3):128-32.

4. Kumar V, Abbas AK, Fausto N, Aster JC. Robbins and Cotran pathologic basis of disease, professional edition e-book.elsevier health sciences; 2014 Aug 27.

5. Perez-Cornago A et al. Prospective investigation of risk factors for prostate cancer in the UK Biobank cohort study.British journal of cancer. 2017;117(10):1562.

6. Steck SE et al. Calcium, magnesium, and whole-milk intakes and high-aggressive prostate cancer in the North Carolina-Louisiana Prostate Cancer Project (PCaP). The American journal of clinical nutrition. 2018;107(5):799-807.

7. Allott EH et al. Saturated fat intake and prostate cancer aggressiveness: results from the population-based North Carolina-Louisiana Prostate Cancer Project. Prostate cancer and prostatic diseases. 2017;20(1):48.

8. Aaron L, Franco OE, Hayward SW. Review of prostate anatomy and embryology and the etiology of benign prostatic hyperplasia. Urologic Clinics. 2016 Aug 1;43(3):279-88.

9. Rosai J. Rosai and Ackerman's surgical pathology e-book. Elsevier Health Sciences; 2011 Jun 20.

10. Bhavsar A, Verma S. Anatomic imaging of the prostate. BioMed research international. 2014
11. Wilson AH. The prostate gland: a review of its anatomy, pathology, and treatment. Jama. 2014 ;312(5):562-.

12. Lee $\mathrm{CH}$, Akin-Olugbade O, Kirschenbaum A. Overview of prostate anatomy, histology, and pathology. Endocrinology and Metabolism Clinics. 2011;40(3):565-75.

13. Coakley FV, Hricak H. Radiologic anatomy of the prostate gland: a clinical approach. Radiologic Clinics of North America. 2000;38(1):15-30.

14. Giannico GA, Arnold SA, Gellert LL, Hameed O. New and emerging diagnostic and prognostic immunohistochemical biomarkers in prostate pathology.Advances in anatomic pathology. 2017;24(1):35-44.

15. Waqas M, Shohab D, Khawaja MA, Masood A, Iqbal MW, Akhter S. Outcome Of Trans Rectal Ultrasound Guided Twelve Core Biopsy Of Prostate For The Detection Of Prostate Cancera Single Center Experience. Journal of Ayub Medical College Abbottabad. 2017;30(1):49-53.

16. Xiao X, Hu R, Deng FM, Shen SS, Yang XJ, Wu CL. Practical applications of immunohistochemistry in the diagnosis of genitourinary tumors. Archives of pathology \& laboratory medicine. 2017;141(9):1181-94.

17. Morlando M, Pelullo CP, Di Giuseppe G. Prostate cancer screening: Knowledge, attitudes and practices in a sample of men in Italy. A survey.PloS one. 2017;12(10):e0186332.

18. Wong $\mathrm{MC}$ et al. Global incidence and mortality for prostate cancer: analysis of temporal patterns and trends in 36 countries. European urology. 2016;70(5):862-74.

19. Carlsson S, Assel M, Sjoberg D, Ulmert D, Hugosson J, Lilja $\mathrm{H}$, Vickers A. Influence of blood prostate specific antigen levels at age 60 on benefits and harms of prostate cancer screening: population based cohort study. Bmj. 2014;348: g2296.

20. Jin J. Screening for Prostate Cancer. Jama. 2018;319(18):1946

21. Duskova K, Vesely S. Prostate Specific Antigen. Current clinical application and future prospects.

22 Diamandis EP. Cancer biomarkers: can we turn recent failures into success?.Journal of the National Cancer Institute. 2010;102(19):1462-7.

23. 9.Lin PY, Cheng KL, McGuffin-Cawley JD, Shieu FS, Samia AC, Gupta S, Cooney M, Thompson CL, Liu CC. Detection of alpha-methylacyl-CoA racemase (AMACR), a biomarker of prostate cancer, in patient blood samples using a nanoparticle electrochemical biosensor. Biosensors. 2012;2(4):377-87.

24. Yamoah K, Johnson MH, Choeurng V, Faisal FA, Yousefi K, Haddad Z, Ross AE, Alshalafa M, Den R, Lal P, Feldman M. Novel biomarker signature that may predict aggressive disease in African American men with prostate cancer. Journal of Clinical Oncology. 2015;33(25):2789.

25. Gurel B et al. NKX3. 1 as a marker of prostatic origin in metastatic tumors. The American journal of surgical pathology. 2010;34(8):1097.

26. Nodouzi V, Nowroozi M, Hashemi M, Javadi G, Mahdian R. Concurrent down-regulation of PTEN and NKX3. 1 expression in Iranian patients with prostate cancer. International braz $\mathrm{j}$ urol. 2015 Oct;41(5):898-905.

27. Bowen C, Zheng T, Gelmann EP. NKX3. 1 suppresses TMPRSS2-ERG gene rearrangement and mediates repair of androgen receptor-induced DNA damage. Cancer research. 2015; 14 :canres-3387 
28. Kurita T, Medina RT, Mills AA, Cunha GR. Role of p63 and basal cells in the prostate. Development. 2004 Oct $15 ; 131(20): 4955-64$

29. Yang K, Wu WM, Chen YC, Lo SH, Liao YC. Np63á Transcriptionally Regulates the Expression of CTEN That Is Associated with Prostate Cell Adhesion. PloS one. 2016;11(1):e0147542.

30. Murphy AB, Akereyeni F, Nyame YA, Guy MC, Martin IK, Hollowell CM, Walker K, Kittles RA, Ahaghotu C. Smoking and prostate cancer in a multi-ethnic sample. The Prostate. 2013;73(14):1518-28.

31. Cao Z, Kyprianou N. Mechanisms navigating the TGF-â pathway in prostate cancer. Asian journal of urology. 2015;2(1):11-8.

32. Barrett CS, Millena AC, Khan SA. TGF-â effects on prostate cancer cell migration and invasion require FosB. The Prostate. 2017;77(1):72-81

33. Mian OY et al. GSTP1 Loss results in accumulation of oxidative DNA base damage and promotes prostate cancer cell survival following exposure to protracted oxidative stress. The Prostate. 2016;76(2):199-206.

34. Martignano $\mathrm{F}$ et al. GSTP1 methylation and protein expression in prostate cancer: diagnostic implications. Disease markers. 2016;2016

35. Burgio SL et al. Chromogranin A predicts outcome in prostate cancer patients treated with abiraterone. Endocrine-related cancer. 2014 Jun 1;21(3):487-93.
36. Erb $\mathrm{HH}$ et al. IL- 6 sensitizes prostate cancer to the anti proliferative effect of IFNá2 through IRF9. Endocrine-related cancer. 2013:ERC-13.

37. Yu SH et al. A paracrine role for IL6 in prostate cancer patients: lack of production by primary or metastatic tumor cells. Cancer immunology research. 2015;3(10):1175-84.

38 Wang L, Ma L, Wang X, Li B, Guo S, Qiao Q. Association of serum EPCA-2 level with prostate cancer in Chinese Han population. International journal of clinical and experimental pathology. 2015;8(8):9397.

39. Pourmand $G$ et al. EPCA2. 22: A Silver Lining for Early Diagnosis of Prostate Cancer. Urology journal. 2016;13(5):2845-8.

40. Jamaspishvili $\mathrm{T}$ et al. Clinical implications of PTEN loss in prostate cancer.Nature Reviews Urology.2018 Feb 20.

41. Adamo $P$ et al. The oncogenic transcription factor ERG represses the transcription of the tumour suppressor gene PTEN in prostate cancer cells. Oncology letters. 2017 Nov 1;14(5):5605-10.

42. Phin S, Moore M, Cotter PD. Genomic rearrangements of PTEN in prostate cancer. Frontiers in oncology. 2013;3:240 